\title{
Cambio climático y adaptación para la región de los Santanderes: percepciones y consideraciones desde el marco legal
}

\author{
Julia Bedoya-Mashuth ${ }^{\star}$, Melva Salazar de Cardona ${ }^{\star \star}$
}

* Médico Veterinario y Zootecnista. Investigadora, grupo de investigaciones en Ciencias Animales, Universidad Cooperativa de Colombia, Bucaramanga, Colombia. Coinvestigadora, Centro de Investigaciones en Medio Ambiente y Desarrollo (CIMAD), Universidad de Manizales, Manizales, Colombia.

Correo electrónico: julia.bedoya@campusucc.edu.co

** Magíster en Medio Ambiente y Desarrollo. Investigadora, Centro de Investigaciones en Medio Ambiente y Desarrollo (CIMAD), Universidad de Manizales, Manizales, Colombia. Correo electrónico: melva2008@hotmail.com

Recibido: 14 de marzo del 2014 Aprobado: 5 de mayo del 2014

Cómo citar este artículo: Julia Bedoya-Mashuth \& Melva Salazar de Cardona. Cambio climático y adaptación para la región de los Santanderes: percepciones y consideraciones desde el marco legal. DIXI. Junio 2014. Pág. 71. doi: http://dx.doi. org/10.16925/di.v16i19.733

\begin{abstract}
Resumen
El cambio climático ha generado importantes efectos sociales, económicos y ambientales en el país, situación que ha llevado a la formulación reciente de políticas nacionales de adaptación, además de la activación y puesta en marcha de diferentes acciones regionales. Este artículo pretende dar respuesta a varios interrogantes relacionados con el conocimiento, las actividades, los presupuestos, la voluntad política, los impactos y otros aspectos referentes a este fenómeno y, específicamente, sobre las posibilidades de adaptación en la región de los Santanderes. Esta investigación, con enfoque empírico-analítico, consideró las variables cambio climático y adaptación, y buscó determinar y analizar la capacidad de adaptación en dicha región. Para lograrlo, fueron consultadas fuentes de información de entidades internacionales, nacionales y departamentales y realizadas entrevistas a tres grupos focales y una encuesta semiestructurada en línea a funcionarios y dependencias de 127 municipios.
\end{abstract}

Palabras claves: adaptación, cambio climático, gestión del riesgo, impactos ambientales, políticas públicas.

Climate Change and Adaptation for the Region of the Santanderes: Perceptions and Considerations from the Legal Framework

\section{Abstract}

Climate change has generated significant social, economic and environmental changes in the country, which has led to the recent formulation of national policies for adaptation, in addition to the activation and implementation of diverse regional actions. This article aims to respond to various questions relating to knowledge, activities, budgets, political will, impacts and other aspects associated with this phenomenon and, specifically, the possibilities for adaptation in the region of the Santanderes. The research, through an empirical-analytical approach, examined such variables as climate change and adaptation and sought to determine and analyze the capacity for adaptation in that region. To do so, information sources from international, national and departmental entities were consulted and interviews were held with three focal groups along with implementation of a semi-structured online survey of officials and agencies in 127 municipalities.

Keywords: adaptation, climate change, risk management, environmental impacts, public policies.

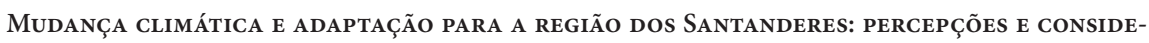
RAÇÕES A PARTIR DO MARCO LEGAL

\section{Resumo}

A mudança climática tem gerado importantes efeitos sociais, econômicos e ambientais no país, situação que tem levado à formulação recente de políticas nacionais de adaptação, além da ativação e a colocação em funcionamento de diferentes ações regionais. Este artigo pretende dar resposta a vários interrogantes relacionados com o conhecimento, as atividades, os pressupostos, a vontade política, os impactos e outros aspectos referentes a esse fenômeno e, especificamente, sobre as possibilidades de adaptação na região dos Santanderes. Esta pesquisa, com enfoque empírico-analítico, considerou as variáveis mudança climática e adaptação, e procurou determinar e analisar a capacidade de adaptação nessa região. Para consegui-lo, foram consultadas fontes de informação de entidades internacionais, nacionais e estaduais, e realizadas entrevistas a três grupos focais e uma enquete semiestruturada on-line a funcionários e dependências de 127 municípios.

Palavras-chave: adaptação, mudança climática, gestão do risco, impactos ambientais, políticas públicas. 


\section{INTRODUCCIÓN ${ }^{1}$}

La propuesta de investigación del macropoyecto estuvo encaminada al estudio del impacto del cambio climático en Colombia. Para su análisis, fueron consideradas las categorías de análisis, adaptación y cambio climático para la región de los Santanderes. Las preguntas de investigación fueron las siguientes: ¿cuál es la capacidad de adaptación de las diversas regiones para afrontar las situaciones derivadas del cambio climático? y ¿cuáles son las posibilidades de adaptación de las regiones al cambio climático?

\section{AnteCEDENTES DEL CAMBio} CLIMÁTICO Y ADAPTACIÓN EN EL CONTEXTO INTERNACIONAL Y NACIONAL

\section{A. Cambio climático}

La preocupación por el cambio climático se percibía desde años atrás. Las Naciones Unidas en su Declaración de Estocolmo sobre el Medio Humano (1972) expresaba, entre sus principios y recomendaciones, la preocupación por las descargas y la contaminación de tal magnitud que no pudieran ser neutralizadas por el mismo medio. Consideraron importante evaluar agentes contaminantes (probabilidad y magnitud) que pudieran generar efectos sobre el clima, y asimismo se recomendó evaluar globalmente el medio ambiente.

En 1988 se celebró, en Canadá, el Congreso de Toronto sobre cambios en la atmósfera, escenario que permitió el establecimiento del Panel Intergubernamental de Expertos sobre el Cambio Climático (IPCC), encargado de analizar la información científica necesaria para abordar el problema del cambio climático y las consecuencias medioambientales y socioeconómicas. Para 1990, se produce el primer informe del IPCC, documento base de la conferencia de las partes para la adopción del Convenio Marco de la Naciones Unidas sobre Cambio Climático en 1992 y el Protocolo de Kioto (1997), ${ }^{2}$

1. Este trabajo hace parte del macroproyecto Estado y prospectiva de las posibilidades de adaptación y mitigación de impacto del cambio climático en diferentes regiones del país, desarrollado por el Centro de Investigaciones en Medio Ambiente y Desarrollo (CIMAD) y la Maestría en Desarrollo Sostenible y Medio Ambiente de la Universidad de Manizales.

2. Grupo Intergubernamental de Expertos sobre el Cambio Climático (IPCC). CAMBIO CLIMÁTICO 2007: INFORME DE SÍNTESIS. OMM Y PNUMA. (2008) coincidiendo en este la Declaración de Río sobre Medio Ambiente y Desarrollo, espacio que reafirma la Declaración de Estocolmo (1972), que expresa también en algunos principios su preocupación y las medidas por tomar en el tema ambiental.

Con el concurso de esfuerzos conjuntos de diferentes países, y dada la preocupación fundada, quedó definido el tratado internacional jurídicamente vinculante de la Convención Marco de la Naciones para el Cambio Climático, reconociendo la problemática, directrices y compromisos en torno al cambio climático a nivel mundial. Colombia, no ajena a esta problemática, aprueba la CMNUCC mediante la Ley 164 de 1994, por tanto hace parte de los 186 países que ya ratificaron el mismo, dando garantía de uno de los acuerdos con mayor respaldo político, dado el número de Gobiernos comprometidos. Por su parte, con la Ley 629 del 2000 se da la aprobación del Protocolo de Kioto de la Convención Marco de las Naciones Unidas sobre Cambio Climático hecho en 1997.

Tomando como referente la definición contemplada en el artículo primero del informe de la Convención Marco de las Naciones Unidas para el Cambio Climático, este se especifica como un "cambio de clima atribuido directa o indirectamente a la actividad humana que altera la composición de la atmósfera mundial y que se suma a la variabilidad natural del clima observada durante períodos de tiempo comparables".

Ahora bien, ese cambio supone efectos adversos, referidos a cambios en el medio ambiente físico o en la biota con consecuencias nocivas significativas en la composición, la capacidad de recuperación o la productividad de los ecosistemas naturales o sujetos a ordenación, o en el funcionamiento de los sistemas socioeconómicos, o en la salud y el bienestar humanos. ${ }^{4}$

Por otro lado, la Convención diferencia entre cambio climático atribuido a actividades humanas, que alteran la composición atmosférica, y la variabilidad climática atribuida a causas naturales. Esta última la relacionan con las variaciones en el estado medio y otros datos estadísti$\cos$ (como las desviaciones típicas, la ocurrencia de fenómenos extremos, etcétera) del clima en todas las escalas temporales y espaciales, más allá de fenómenos meteorológicos determinados. Igualmente, aclaran que esa variabilidad se puede presentar, ya sea por procesos internos naturales dentro del sistema climático (variabilidad inter-

\footnotetext{
3. Naciones Unidas. Convención Marco de las Naciones Unidas para el Cambio Climático. (1992). Disponible en: http://unfccc.int/ resource/docs/convkp/ convsp.pdf

4. Id.
} 
na), ya sea por variaciones en los forzamientos externos antropogénicos (variabilidad externa). ${ }^{5}$

La Convención también menciona que la no linealidad del sistema climático puede llevar a un rápido cambio climático, lo que se denomina a veces fenómenos repentinos o imaginables o incluso sorpresivos, es decir, pueden suceder sin que se esperen como consecuencia del forzamiento fuerte y rápidamente cambiante de un sistema no lineal. Ya en el cuarto informe del IPCC, se enuncia que el cambio climático puede deberse a procesos internos naturales, a forzamientos externos o por cambios antropógenos persistentes de la composición de la atmósfera o del uso de la tierra. ${ }^{6}$

Es de resaltar, como lo señala el Instituto de Hidrología, Meteorología y Estudios Ambientales (IDEAM), que el cambio climático se debe, en parte, al incremento de las emisiones de gases de efecto invernadero (GEI), aclarando que existe una diferencia entre variabilidad climática y cambio climático. La primera aparece cuando con cierta frecuencia un fenómeno genera un comportamiento anormal del clima, pero es un fenómeno temporal y transitorio, mientras que el cambio climático se asocia a un proceso que no es temporal y que puede verificarse en el tiempo revisando datos climáticos. El IDEAm señala que el IPCC describe un enfoque integrado del cambio climático, teniendo en cuenta la dinámica del ciclo completo de causas y efectos interrelacionados en todos los sectores afectados. Dicho marco de evaluación integrado de cambio climático por causas antropogénicas considera estos aspectos: emisiones y concentraciones de GEI, cambio climático, impactos en los sistemas humanos y naturales, aspectos relacionados con las vías socioeconómicas de desarrollo. Asimismo, señala los puntos de adaptación y mitigación del cuadrante integrado. ${ }^{7}$

En Colombia, desde años atrás, ya se había previsto sobre este fenómeno y, de igual forma, se había concluido que en el país se tiene poco control sobre sus causas; por tanto, habría que dedicarle un gran esfuerzo y prepararse para sus impactos. ${ }^{8}$ En el 2003, con la presenta-

5. Grupo Intergubernamental de Expertos Sobre el Cambio Climático (IPCC). CAMBio CLIMÁtico 2001: INFORME DE SÍNTESIS. RESUMEN PARA RESPONSABLES DE POLÍ́TICAS. (2001).

6. Grupo Intergubernamental de Expertos sobre el Cambio Climático (ipCC). Segunda eVAluación: CAMbio Climático. PNUMA. (1995).

7. Id.

8. Carlos Costa Posada. (Noviembre de 2007). La adaptación al cambio climático en Colombia. Revista de Ingenierías 26, Noviembre del 2007. Págs. 74-80. [Recuperado el 12 de agosto de 2013 de http://www. scielo.org.co/pdf/ring/n26/n26a10.pdf] ción del documento Conpes 3242, se esperaba promover la incursión de Colombia en el mercado internacional de reducciones verificada de emisiones de GEI. Por tanto, la intención de la estrategia planteada en el documento se enfocó en la venta de este servicio ambiental: de mitigación del cambio climático.

Para el 2012, el Ministerio de Ambiente y Desarrollo Sostenible plantea en el Plan Nacional de Cambio Climático su incorporación en políticas, planes, programas y entes territoriales. ${ }^{9}$ Dado que el cambio climático ya es aceptado como una realidad, y de acuerdo con el Gobierno, por los significativos efectos en el desarrollo socioeconómico, se ha formulado dicho plan, en la búsqueda de disminuir los riesgos e impactos socioeconómicos asociados al cambio y a la variabilidad climática. En este plan se recomienda que el cambio climático sea incorporado en los sectores y los entes territoriales dentro de sus políticas, planes, programas y proyectos. ${ }^{10}$ Para el 2013, se destaca, en el marco de dicho plan, la hoja de ruta para la formulación de planes sectoriales y territoriales.

\section{B. Adaptación al cambio climático}

El IPCC, en su informe de síntesis Cambio climático 2007, define la adaptación como el ajuste en sistemas humanos o naturales en respuesta a los estímulos climáticos, que minimizan el posible daño ocasionado por estos cambios o que potencian sus efectos positivos. Se debe tener en cuenta que las estrategias de adaptación están propuestas para responder a las necesidades, oportunidades y prioridades identificadas en la evaluación de la vulnerabilidad de los sectores productivos, los recursos naturales y la población.

Se pueden diferenciar varios tipos de adaptación, entre ellos, la preventiva y la reactiva, la pública y privada, o la autónoma y la planificada, siendo esta última de interés para esta revisión, caracterizada por cinco líneas estratégicas, las cuales fueron propuestas para servir como guías generales en la formulación de planes de adaptación en los diferentes sectores y territorios: concientización sobre el cambio climático, generar información y conocimiento para medir el riesgo climático, planificar el uso del territorio, implementar acciones de adaptación y fortalecer la capacidad de reacción (DNP,

9. Oficina Asesora de Planeación. Informe De Gestión 2011. Ministerio de Ambiente y Desarrollo Sostenible. (2012). [De aquí en adelante Oficina Asesora]

10. Id. 
MADS, IDEAM y UNGRD). De igual forma, la capacidad de adaptación se relaciona con la capacidad de un sistema para ajustarse al cambio climático (incluida la variabilidad climática y los cambios extremos), para moderar los daños potenciales, aprovechar las consecuencias positivas, o soportar las consecuencias negativas. ${ }^{11} \mathrm{El}$ IPCC, en su informe 2007, afirma que la capacidad de adaptación al cambio climático va depender de las condiciones socioeconómicas, medioambientales, de la disponibilidad de información, así como de tecnología. A pesar de ser concluyente en que la adaptación será esencial y beneficiosa, reconoce limitaciones financieras, tecnológicas, cognitivas, comportamentales, políticas, sociales, institucionales y culturales, que seguramente limitan tanto la aplicabilidad como la efectividad de las medidas de adaptación. El IPCC menciona que las sociedades con una alta capacidad adaptativa son vulnerables al cambio climático, a la variabilidad y a los extremos climáticos. ${ }^{12}$

En Colombia, la formulación del Plan Nacional de Adaptación al Cambio Climático viene de la mano con lo propuesto en el Conpes 3700 . Aquí se define la estrategia institucional para la articulación de políticas y acciones sobre cambio climático en el país, en que se destaca la necesidad de entender y actuar frente al cambio climático como una problemática de desarrollo económico y social, condiciones que hasta el momento no eran estimadas. Por ello, se plantea la necesidad de integrar la problemática de cambio climático en la planificación de los sectores y territorios, por tanto se espera que las autoridades, sectores y territorios, al incluir estas variables en sus planes, utilicen los recursos de forma adecuada, reduzcan la exposición y sensibilidad al riesgo y amplíen la capacidad de respuesta. Así se reconoce la necesidad inmediata de emprender medidas adecuadas de adaptación y mitigación para reducir la vulnerabilidad y, por tanto, favorecer un escenario futuro de desarrollo económico sostenible para el país.

De igual forma, se debe destacar que el Conpes 3700 pretende atender dos de los más grandes desafíos que representa en Colombia el cambio climático: la coordinación intersectorial y la disponibilidad de información para la toma de decisiones (estrategia institucional). ${ }^{13}$

11. Véase IPCC, supra, nota 6.

12. Véase IPCC, supra, nota 5.

13. Véase Oficina Asesora, supra, nota 9.
C. Vulnerabilidad y su relación con la sensibilidad y la adaptación

La vulnerabilidad, definida como el grado en que el cambio del clima puede ser perjudicial o nocivo para un sistema, no sólo depende de la sensibilidad del sistema, sino también de su capacidad para adaptarse a nuevas condiciones climáticas. Tanto la magnitud como la velocidad del cambio climático son importantes para determinar la sensibilidad, la adaptabilidad y la vulnerabilidad de un sistema. ${ }^{14}$ Para el 2001, para su informe de síntesis, el IPCC señala la vulnerabilidad como el nivel al que un sistema es susceptible, o no es capaz de soportar, los efectos adversos del cambio climático, incluidos la variabilidad climática y los fenómenos extremos. Para el 2008, en Cambio climático 2007, ajustan este concepto al grado de susceptibilidad o de incapacidad de un sistema para afrontar los efectos adversos del cambio climático y, en particular, la variabilidad del clima y los fenómenos extremos.

En cuanto a la sensibilidad, se establece como el grado en que un sistema reacciona a un cambio en las condiciones climáticas. El IPCC concibe, en su Segunda evaluación: cambio climático de 1995, la sensibilidad del clima como el cambio a largo plazo (equilibrio) de la temperatura superficial media global como consecuencia de una duplicación de la concentración de $\mathrm{CO}_{2}$ equivalente en la atmósfera. También se puede entender como el grado en que un sistema resulta afectado, ya sea positiva o negativamente, por la variabilidad o el cambio climático. El efecto puede ser directo o indirecto. ${ }^{15}$

Finalmente, a la vulnerabilidad, se le suman dos componentes: la sensibilidad y capacidad de adaptación. La primera referida a la predisposición física del ser humano, la infraestructura o un ecosistema de ser afectados por una amenaza, debido a las condiciones de contexto e intrínsecas que potencian el efecto de la amenaza, es decir, mide la debilidad del sistema. La segunda se entiende como la capacidad de un sistema y de sus partes de anticipar, absorber, acomodar o recuperarse de los efectos de un disturbio, de una forma oportuna y eficiente y, por tanto, mide la capacidad del sistema de afrontar y recuperarse ante un evento. Adicionalmente, se espera que, con la gestión del riesgo de desastres y la adaptación al cambio climático como estrategias complementarias, se disminuya

14. Véase IPCC, supra, nota 6.

15. Véase Grupo Intergubernamental de Expertos Sobre el Cambio Climático (IPCC). Anexo B: glosario de términos. CAMBIo Climático 2001: INFORME DE SÍNTESIS. RESUMEN PARA RESPONSABLES DE POLÍticAs. (2001). [En línea]. Disponible en: http://www.ipcc.ch/ pdf/glossary/taripcc-terms-sp.pdf [Consultado el 18 de mayo de 2011]. 
el riesgo climático y sus impactos socioeconómicos. Tanto así que el Gobierno ha anunciado que, al terminar el periodo, se espera contar con 142 planes de ordenamiento del riesgo frente al cambio climático.

\section{Gestión del riesgo de desastres y el cambio climático}

Con la adopción de la Política Nacional de Gestión de Riesgo de Desastres, se pretende mejorar la calidad de vida de las comunidades en riesgo, por tanto, considera la planificación del desarrollo seguro y la gestión ambiental territorial sostenible. La estructura de esta política busca precisamente contar con diferentes mecanismos (políticas, estrategias, planes, programas, regulaciones, instrumentos, medidas y acciones) para afrontar eventos naturales, entre ellos, el cambio climático, los cuales quedaron en evidencia por la grave calamidad pública presentada en la ola invernal del 2010, dejando ver la debilidad del Sistema Nacional para la Atención y Prevención de Desastres, lo que llevó a la formulación de la declaratoria de emergencia social, económica, ambiental. En esta política están definidas las directrices para la actuación a partir del conocimiento, la reducción del riesgo y la respuesta en caso de desastre.

\section{E. Políticas y estrategias frente al cambio climático en la región}

En el orden regional, el nodo Regional Norandino de Cambio Climático, grupo regional, interinstitucional e interdisciplinario, conformado por instituciones públicas y privadas del orden local, departamental y regional, que trabajan articuladamente bajo la coordinación del Ministerio de Medio Ambiente y Desarrollo Sostenible, que busca contribuir a la formulación, promoción, articulación y desarrollo de políticas, lineamientos y estrategias interinstitucionales del cambio climático, ${ }^{16}$ en los dos últimos años ha contado con una nutrida actividad, con participación del Ministerio de Ambiente y Desarrollo Sostenible, la Corporación Autónoma de Santander, la Corporación Autónoma para la Defensa de la Meseta de Bucaramanga, la Corporación Autónoma Regional de la Frontera Nororiental, las Instituciones de Educación Superior, las organizaciones no gubernamentales, entre otros.

16. Véase Oficina Asesora, supra, nota 9.
El Nodo Norandino fue constituido en el 2010 cuando adelantó el taller "Análisis y fortalecimiento de estructura institucional y del plan de acción del nodo regional de cambio climático Norandino", en el cual se constituyó y quedó formulado su plan de acción (octubre 2010). Para el 2012, se destaca una reunión, convocada por el MinAmbiente y con la participación de la WWF Colombia, la cual centró su agenda en analizar y definir una estructura institucional - arreglos entre las entidades que facilitaran la gestión de medidas de mitigación y adaptación al cambio climático- y buscó también integrar el nodo regional de cambio climático Norandino, plantear mecanismos operativos para su funcionamiento y avanzar en la consolidación del Plan de Acción del nodo.

En el departamento, los planes de desarrollo de la región han incorporado aspectos sobre el mismo: en el Plan de Desarrollo de Santander (2012-2015) se proponen unas líneas de acción en torno a la adaptación al cambio climático. El Plan de Desarrollo de Norte de Santander registra, en el eje para el desarrollo territorial "Un norte sostenible”, dentro del programa Gestión Ambiental Participativa, algunos proyectos que abordan el calentamiento global y promueven una alianza para la consolidación del nodo de adaptación al cambio climático.

\section{F. Estudios sobre cambio climático en la región de los Santanderes}

A nivel local, un estudio realizado en la región de Betulia, San Vicente de Chucuri, Zapatoca, Girón y Lebrija, buscó conocer percepciones sobre variabilidad climática y cambio climático en la población de la región objeto de estudio mediante aplicación de encuestas a 487 campesinos. Dentro de las conclusiones del estudio se encontró:

Se identificó que las estrategias de adaptación espontánea
no son reconocidas como tal entre la gente, puesto que
las diferentes acciones de cambio y/o ajustes han surgido
y surgen de manera empírica y hacen parte de la cotidia-
nidad. Frente a la documentación técnica de las percep-
ciones sobre la alteración de las variables climatológicas,
es importante concluir que desde el punto de vista cien-
tífico se seguirán encontrando "concordancias" entre lo
objetivo y lo subjetivo, ya que los saberes locales - no
medibles- establecen otra forma de conocimiento que
se justifica con base en la experiencia y por tal razón, son
conocimientos válidos, verificables y con credibilidad. ${ }^{17}$

17. María Carolina Pinilla, Andrés Rueda, Carlos Pinzón y Javier Sánchez. Percepciones sobre los fenómenos de variabilidad climática y cambio climático entre campesinos del centro de Santander, Colombia. Аmbiente 


\section{MATERIALES Y MÉTODOS}

\section{A. Localización}

La unidad de trabajo comprendió la región de los Santanderes compuesta por los 87 municipios del departamento Santander y 40 de Norte de Santander. El primero localizado entre la latitud norte $05^{\circ} 42^{\prime}$ y $08^{\circ}$ con longitud entre $72^{\circ} 26^{\prime}$ y $74^{\circ} 32^{\prime}$, con una extensión de $30.537 \mathrm{~km}^{2},{ }^{18}$ altitud que va desde 100 a $4000 \mathrm{msnm}$, temperatura promedio de 9 a $32{ }^{\circ} \mathrm{C}$ y $\mathrm{HR}$ de 40 a $38 \% .^{19}$ El segundo situado en el extremo nororiental de Colombia, en coordenadas $6^{\circ} 58^{\prime}$ de latitud norte y $72^{\circ} 03^{\prime}$ y $73^{\circ} 35^{\prime}$ de longitud occidental, con una extensión de $21.987 \mathrm{~km}^{2},{ }^{20} 100-4500 \mathrm{msnm}$ la altitud, temperaturas que oscilan de los 3 a los $30^{\circ} \mathrm{C}$ y HR de 60 a 92\% (figura 1).

\section{B. Población y muestra}

Se contó con diferentes informantes claves de los municipios de Santander y Norte de Santander. Inicialmente, como una primera aproximación piloto, se adelantó una entrevista a seis funcionarios vinculados a diferentes entidades. Posteriormente, se contó con la participación de tres grupos focales (funcionarios de entidades públicas y privadas y comunidad vulnerable). Y por último, se adelantó una encuesta en línea a los $127 \mathrm{mu}$ nicipios de Santander y Norte de Santander. Dentro de los actores, se destaca la participación de alcaldes, secretarios de planeación, autoridades ambientales, profesionales y técnicos ambientales y contratistas.

\section{Metodología}

\section{Fase empírico-analítica y descriptiva}

Considerando las categorías de análisis cambio climático y adaptación. En esta fase, se establecieron dos momentos: el primero consideró una investigación do-

y Desarrollo 31. Diciembre de 2012. At. 25.

18. http://www.santander.gov.co/gobernacion_mm/estatica/images/destacados/pag/pdd/index.php?sec=1 (Mayo 25 de 2012).

19. http://www.santander.gov.co/gobernacion_mm/estatica/images/destacados/pag/pdd/index.php?sec=1 (Mayo 25 de 2012).

20. Gobernación de Norte de Santander. Información general: norte de Santander. [En línea] Disponible en http://www.nortedesantander.gov. co/infgeneral.php [Consultado el 24 de mayo de 2012]. cumental a partir de fuentes primarias y secundarias del MinAmbiente, el IDEAM, las corporaciones autónomas regionales y entidades del orden regional, y los planes de desarrollo, departamentales y municipales.

El segundo consistió en aplicación de encuestas semiestructuradas en línea:

- La primera se adelantó a un grupo de informantes claves, vinculados a diferentes instituciones y empresas de los Santanderes (Empopamplona, S. A. E. S. P., Cormagdalena, Alcaldía de Ocaña, Fundación Natura, Secretaría de Agricultura de Santander, Corporación Autónoma para la Defensa de Bucaramanga.

- La segunda encuesta en línea consideró diferentes niveles jerárquicos y dependencias de nivel alto (alcaldes, gobernadores, concejales, diputados, directores de corporaciones, secretarios de despacho, directores de unidades municipales de asistencia técnica (Umata), directores de grupos de investigación, directores de nodos regionales, etcétera), medio (asistentes de secretarías, investigadores, subdirectores de corporación y nodos regionales, consultores, profesionales universitarios, asistentes, etcétera), bajo (auxiliares, secretarios, técnicos de Umata, practicantes, etcétera) y sus dependencias de los 87 municipios del departamento de Santander encuestados. En esta segunda consulta, se obtuvo respuesta del 55\%, mientras que para Norte de Santander (40 municipios) respondió el 82,5\%.

- Las encuestas aplicadas consideraron preguntas de percepción relacionadas con el cambio climático y adaptación a este. En cuanto al número de encuestas diligenciadas teniendo en cuenta la codificación según cargo, se encontró que el $23,6 \%$ de los encuestados correspondió a niveles altos, el $37,2 \%$ a niveles medios y el 39,09\% a niveles bajos. En cuanto a las dependencias que participaron en la encuesta en línea, se encontró un $48,2 \%$ de dependencias altas, un $39,09 \%$ de dependencias medias y un $11,8 \%$ de dependencias bajas.

\section{La fase hermenéutica}

Se realizó a partir de diálogos direccionados a través de entrevistas con informantes claves de la región y con grupos focales. El primer grupo con la participación de funcionarios de Parques Naturales Nacionales, la Fundación Corambiente y la Oficina de Gestión de Riesgo 
de la Alcaldía de Bucaramanga. Con el grupo focal, se adelantó mediante un foro virtual con la Corporación Autónoma Regional de la Frontera Nororiental (Corponor), la Alcaldía de Ocaña por Norte de Santander y la Fundación Corambiente por Santander.

\section{RESUlTADOS Y ANÁLISIS}

Los diferentes actores sociales consultados dieron información pertinente a esta temática, a través de los instrumentos arriba mencionados.

Inicialmente, se consultó a seis profesionales de diferentes entidades de Santander y Norte de Santander acerca de las razones para abordar el tema del cambio climático en sus regiones. Todos estuvieron de acuerdo en destacar la importancia de este abordaje y subrayaron la afectación en los ecosistemas y los recursos hídricos, las lluvias y las sequías, la alteración en la producción agrícola, las afectaciones en la salud y en las viviendas.

Estos juicios coinciden con las razones que plantea el Gobierno para promover en el país el Plan Nacional de Adaptación al Cambio Climático, teniendo en cuenta el riesgo y los impactos en el desarrollo social y económico, así como la importancia de proponer acciones de adaptación y mitigación en lo local, formular planes ambientales y de desarrollo, que orienten las decisiones frente a la variación de la precipitación, la temperatura y la variabilidad climática.

Los profesionales encuestados, vinculados a organizaciones privadas y públicas, manifiestan que han abordado el tema del cambio climático en sus empresas mediante estrategias que van desde medidas de mitigación del impacto, basadas en alternativas sostenibles en el manejo de residuos, buscando reducir la emisión de gas metano, hasta la revisión de factores meteorológicos y aspectos socioculturales en el área de influencia de la empresa. Esta revisión se ha realizado a través de estudios sobre captura de carbono en bosques, medición de emisiones y mercado de bonos de carbono. También se encontraron propuestas alternativas de adaptación a las comunidades, participación en la formulación de planes de ordenamiento, manejo de cuencas, formulación de proyectos locales relacionados con producción sostenible (sistemas agroforestales y silvopastoriles), desarrollo de proyectos MDL (mecanismo de desarrollo limpio), capacitación y sensibilización a la comunidad. De los anteriores planteamientos, se percibe conciencia frente a esta nueva realidad; pareciera que las entidades públicas y privadas consultadas en este primer acercamiento han intentado ser diligentes frente a las proyecciones del cambio climático, pues registran participación en la formulación, revisión y aplicación de estrategias, tal como lo denotan en algunos de los programas presentados, lo que significa que han incorporado en sus acciones los lineamientos que, desde el marco nacional, se han venido formulando en los últimos años.

Las instituciones manifiestan que existe una articulación positiva con otras entidades y con comunidades vulnerables al cambio climático, lo que les ha permitido compartir experiencias y, por tanto, mejorar el conocimiento en el tema y lograr la sensibilización en actores regionales, resaltando la importancia de contar con aliados estratégicos tanto locales como internacionales.

Dentro de las estrategias de articulación, destacan hacer parte de mesas de trabajo de cambio climático, como el Nodo Regional Norandino de Cambio Climático, redes latinoamericanas, mesas de trabajo en educación, participación en proyectos de investigación interinstitucionales con organizaciones internacionales y empresas privadas, principalmente para la gestión de fondos externos. Asimismo, han adelantado convenios con universidades de la región y manifiestan que han trabajado con entes territoriales en la búsqueda de ayudar a la comunidad vulnerable.

Ya en el ámbito nacional, el Gobierno ha sumado esfuerzos para involucrar a diferentes ministerios, teniendo en cuenta que la anterior visión de la problemática del cambio climático se circunscribía a lo ambiental $y$, por ende, no era considerado este fenómeno y su proyección desde el desarrollo económico y social; consideraciones que quedaron claramente expuestas en las pasadas olas invernales del 2010 y del 2011. Respondiendo a estas claras intenciones, se crea un sistema de articulación institucional en el marco de la "Estrategia institucional para la articulación de políticas y acciones en materia de cambio climático." ${ }^{21}$

En cuanto a las organizaciones gubernamentales que están trabajando en el tema de cambio climático,

21. Departamento Nacional de Planeación. Documento Conpes 3700. ESTRATEGIA INSTITUCIONAL PARA LA ARTICULACIÓN DE POLÍTICAS Y aCCiOnes en materia de CAmbio Climático en Colombia. (2011). [De aquí en adelante Departamento]. Véase también Michael Comstock, Ignacio Santelices y Anmol Vanamali. CASO DE ESTUdiO: PROCESO NACional de CAmbio climático en Colombia. Center for Clean Air Policy. (2012). 
los encuestados manifiestan el compromiso de entidades como la Corporación Autónoma para Defensa de la Meseta de Bucaramanga, la Corporación Autónoma de Santander y la Corporación Autónoma Regional de la Frontera Nororiental, el IDEAM, el Instituto de Investigaciones Marinas y Costeras (Invemar), el Ministerio de Ambiente y Desarrollo Sostenible, la Gobernación de Santander, Colombia Humanitaria, la Unidad Nacional de Gestión del Riesgo de Desastres (UNGRD). Asimismo, dan a conocer una serie de programas y acciones que vienen adelantando las entidades en el tema del cambio climático. En lo local, destacan el desarrollo de talleres departamentales dirigidos a entidades públicas, privadas, nivel productivo y sociedad civil, proyectos piloto con comunidades indígenas y campesinas y desarrollo de proyectos de mitigación y adaptación.

En el orden nacional, desarrollo de políticas nacionales de cambio climático, estudios de evaluación y modelación de escenarios de cambio climático, proyectos de Mecanismo de Desarrollo Limpio (MDL), el Programa de Reducción de Emisiones de Carbono causadas por la Deforestación y la Degradación de los Bosques (REDD y REDD+), estudios de vulnerabilidad y escenarios IPCC. De acuerdo con lo anterior, reconocen los importantes avances en el país en el marco institucional, el cual busca fortalecer su capacidad. Tal como se refleja en la nueva estructura del Sistema Nacional de Cambio Climático, como lo señala el Conpes 3700 del 2011, lo que se busca es generar ordenación fuerte y eficaz, intentando, entre otras cosas, transferir responsabilidades directas en cabeza de los sectores económicos y los territorios para enfrentar este fenómeno. Finalmente, no se evidencia en el grupo conocimiento sobre un diagnóstico o avances que den cuenta de la capacidad de adaptación frente al cambio climático en la región.

Para la segunda encuesta en línea, dirigida a funcionarios de diferentes niveles jerárquicos y dependencias de los 127 municipios de la región de los Santanderes, se encontró para la pregunta orientada a determinar si las actividades realizadas en las diferentes dependencias estaban articuladas con las políticas relacionadas con el cambio climático establecidas en el рот о Plan de Desarrollo Local, un 83,1 у 77\% para Santander y Norte de Santander, respectivamente, de respuestas afirmativas, las cuales describían, entre las actividades ejercidas la reforestación, el manejo de licencias de impacto ambiental, educación y concientización, reducción de emisiones de gases de efecto invernadero, uso racional del agua, obras de infraes- tructura, programas de prevención y atención a los desastres, plan de desarrollo, plan de acción, estudio de amenazas y riesgo, implementación de tecnologías con sostenibilidad ambiental, control de recursos hídricos, entre otros. De acuerdo con lo anterior, puede afirmarse que las instituciones están incluyendo en sus agendas los lineamientos nacionales sobre cambio climático.

En cuanto a la percepción sobre los impactos generados por el cambio climático en la región, las respuestas no difieren de los aspectos mencionados. ${ }^{22}$ Las percepciones variaron entre aumento de la temperatura, cambios en la producción agrícola, fuertes olas de calor, variabilidad del clima, sequía o inundaciones, según el caso, inestabilidad económica, deterioro de infraestructura, deterioro progresivo de los recursos naturales, deslizamiento, desplazados, escases de agua. Es de anotar que las percepciones en cuanto a sequías e inundaciones se asocian a los principales desastres identificados en el país a causa de las variaciones del clima, reportando en un $90 \%$ emergencias relacionadas con fenómenos hidroclimatológicos y otros (1998-2011).

Se destaca para Santander inestabilidad económica a causa de sequías e inundaciones; en lo social, destrucción de vías; en la parte ambiental, afectación y deterioro de bosques por incendios, poca atención a las políticas de protección al medio ambiente, y en lo cultural, nuevas formas de vivir.

Para Norte de Santander, el efecto económico se ve representado por pérdidas de vivienda y suelos productivos, disminución en los ingresos, gastos en infraestructura. En lo social, referencian afectaciones también por pérdida de vivienda, deterioro de la calidad de vida, desempleo, desplazamiento y damnificados. En lo ambiental, por contaminación, incendios forestales, erosiones, deslizamientos e inundaciones. En lo político, se percibe incredulidad en las políticas nacionales y locales de la gestión de riesgo, desgaste político. Y, finalmente, en lo cultural, modificación de prácticas culturales, hábitos y la forma de vestir, rechazo a la adopción de nuevas tecnologías, cambios por la influencia del turismo.

Frente al conocimiento de experiencias significativas evidenciadoras de acciones locales para la adaptación al cambio climático en los dos departamentos, fue notable el desconocimiento por parte de los encuestados. Sin embargo, fueron mencionados proyectos relacionados con energía alternativa y procesos de reforestación, más vinculados a aspectos de mitigación que

22. Véase Naciones Unidas, supra, nota 3. 
a cuestiones de adaptación propiamente dicha. Finalmente, no se evidencian en el registro los lineamientos que, desde el Plan Nacional de Adaptación al Cambio Climático, plantean desde lo local con enfoque participativo y atendiendo prioridades de los territorios como son creación de capacidades, participación comunitaria, implementación local, generación de información y reconocimiento del saber ancestral..$^{23}$

Frente a la efectividad de las acciones promovidas para la adaptación al cambio climático, fue calificada como mediana su efectividad en el 67,6\% para Santander y en el 53,8\% para Norte de Santander; sin embargo, como se mencionó, la confusión conceptual no permite sacar conclusiones valederas.

El $62 \%$ y el $61,5 \%$, respectivamente, para Santander y Norte de Santander, respondió afirmativamente frente al conocimiento de planes de contingencia en su localidad para enfrentar los efectos adversos del cambio climático, resaltando obras y acciones de protección para los ciudadanos, planes de precaución, plan de gestión de riesgo. De acuerdo con lo anterior, se perciben debilidades para asumir la renovada Política Nacional de Gestión de Riesgo, contenida en la Ley Sistema Nacional de Gestión de Riesgos, que espera fortalecer los instrumentos de coordinación con las entidades territoriales, y cobra importancia, porque ello supone "el reconocimiento de las ventajas al anticipar la ocurrencia de desastres y de fortalecer las comunidades expuestas", considerando que con la pasada ola invernal en el 2010 quedaron expuestas las debilidades del Sistema Nacional para la Atención y Prevención de Desastres ${ }^{24}$ y, por tanto, se espera que los municipios estén asumiendo este cambio; cosa que no se evidencia en el resultado arrojado para esta pregunta. Sumado a que la incorporación de gestión del riesgo al cambio climático es una prioridad del Plan Nacional de Adaptación al Cambio Climático y se articula con la estrategia institucional planteada en el Conpes $3700 .{ }^{25}$

La voluntad política representada en los lineamientos de los últimos gobernantes locales no es calificada de manera positiva, pues consideran que dichos

23. Departamento Nacional de Planeación. Plan Nacional de Adaptación al Cambio Climático: lineamientos y agenda para la adaptación al Cambio Climático en Colombia. Departamento Nacional de Planeación. (2011).

24. Ley 1523 de 2012. Por la cual se adopta la política nacional de gestión del riesgo de desastres y se establece el Sistema Nacional de Gestión del Riesgo de Desastres y se dictan otras disposiciones. Abril 24 de 2012. DO N. ${ }^{\circ} 48411$.

25. Véase Departamento, supra, nota 23. lineamientos no han aportado para que las entidades y la comunidad en general tomen medidas tendientes a cambiar hábitos y estilos de vida para minimizar los efectos del cambio climático, en un 60,6\% para Santander y en un 79,5\% para Norte de Santander. Lo anterior claramente denota, entre otros, falta de aplicación de los lineamientos consignados en la segunda comunicación nacional ante la Convención Marco de las $\mathrm{Na}$ ciones Unidas sobre Cambio Climático, en su estrategia seis: "Educación, formación y sensibilización a públicos". Aunque en la comunicación resaltan avances en este sentido, es importante que desde lo local se promuevan estas iniciativas.

En general, se puede inferir que las políticas locales no están articuladas con las directrices nacionales, porque, a pesar de contar con estrategias trazadas en el Conpes 3700 del 2011, cuya prioridad precisamente es integrar dentro de la planificación de los territorios y sectores la problemática del cambio climático, y de esta manera iniciar medidas convenientes de adaptación y mitigación, no han sido del todo apropiadas por los gobernantes, quienes plantean como estrategia institucional "crear un marco institucional fuerte y eficaz, con el fin de transferir responsabilidades directas en cabeza de los sectores económicos y los territorios para enfrentar este fenómeno". Tal como lo plantea el documento,

[la] institucionalidad ayudará a coordinar y articular las iniciativas existentes en la materia, guiará el desarrollo de nuevas iniciativas, y hará que los sectores y los territorios internalicen la problemática para que generen sus propias estrategias de adaptación y mitigación sobre unas directrices comunes y claras. ${ }^{26}$

Lo anterior expone un factor de vulnerabilidad desde el componente social, que por sus características podría generar mayor exposición al riesgo, al no contar para este caso con una articulación clara y un trabajo coordinado con los entes nacionales. ${ }^{27}$ Igualmente, los lineamientos del Conpes 3700 registran la gestión para el diseño y elaboración de guías metodológicas, las cuales dan la pauta para la inclusión de variables de cambio climático en las herramientas de planificación territorial y ambiental del país. ${ }^{28}$

\footnotetext{
26. Id.

27. Gustavo Wilches-Chaux. EsE ocÉANO DE AIRE EN QUE VIVIMOS. Origen, EVOlUCión, ESTAdo ACTUAL y FUtUros posibles de LA ATMósfera terrestre. La Silueta Ediciones. (2008).

28. Véase Oficina Asesora, supra, nota 9.
} 
El 70,4 y el 80,6\% para Santander y Norte de Santander manifiesta que el clima ha modificado las costumbres de la comunidad de su localidad en los últimos años, lo cual se evidencia en la modificación de las prendas de vestir, que buscan mayor confort. Por otro lado, han iniciado mecanismos para proteger las fuentes de agua, mediante la cultura de reciclaje, evitando arrojar residuos a las fuentes. Se percibe incertidumbre en cuanto a la forma de cultivar y producción de sus cosechas, lo que se traduce en impactos sobre la agricultura. En el estudio presentado por Pinilla et al., ${ }^{29}$ se encuentra que un 36\% de los encuestados tiene percepciones positivas sobre la variabilidad del clima, relacionadas con la producción de cosechas, ya que afirman que les ha permitido cultivar nuevos productos, ${ }^{30}$ lo que denota una posible oportunidad de revisar hasta dónde es factible aprovechar esta circunstancia, para examinar nuevos escenarios de producción local.

Finalmente, las percepciones recogidas del grupo focal, conformado principalmente por funcionarios de entidades públicas, sobre las posibilidades de adaptación al cambio climático (impactos y formas de prepararse al cambio climático) permiten concluir que para algunos de ellos el término cambio climático es relativamente nuevo. Consideran que los trabajos adelantados son desarticulados, y son las actividades antrópicas las que han propiciado la modificación del clima, siendo el cambio climático una materialización de un riesgo. Por otro lado, sugieren que una forma de generar mecanismos de adaptación en los desarrollos urbanos es interviniendo y reglamentando, evitando renunciar a las áreas de reserva y reforzando lo ya existente. Además, mencionan que no se evidencia una real voluntad política para adoptar medidas de adaptación. Esta percepción revela grandes debilidades en los canales de comunicación y articulación con los entes territoriales, en el entendido de que la adaptación es local, participativa y se orienta atendiendo a las prioridades de los territorios, tal como se puntualiza en el Plan Nacional de Adaptación al Cambio Climático, que establece claramente unas líneas estratégicas para una adaptación planificada: concientización sobre el cambio climático, generar información y conocimiento para medir el riesgo climático, planificar el uso del territorio, implementar acciones de adaptación y fortalecer capacidad de reacción.

29. Véase Pinilla et al., supra, nota 17.

30. Id.
Por otro lado, los trabajos que vienen realizando algunas ONG en Santander sobre adaptación al cambio climático, lo enfocan en un modelo de desarrollo sostenible con agricultura orgánica, protección de cuencas, organización social, rescate de la economía campesina. Han trabajado con algunas organizaciones sociales en Norte de Santander, donde han logrado generar impacto en las políticas públicas. Manifiestan preocupación frente al cambio climático y sus impactos en comunidades, principalmente por explotación minera, dado que son políticas de Estado. Finalmente, reconocen que las mismas comunidades indígenas y campesinas protegen y cuidan áreas protegidas. Si bien se evidencian claras estrategias de adaptación al cambio climático desde un enfoque local, es importante revisar esfuerzos locales integrados a los lineamientos nacionales, teniendo en cuenta que, dentro de las medidas trazadas para el sector agropecuario, se conformó la Red Interinstitucional de Cambio Climático y Seguridad Alimentaria, la cual describe metodologías comunes, desarrollo de indicadores para determinar vulnerabilidad y adaptación de sistemas de producción al cambio climático con participación del MinAgricultura y Corpoica. De hecho, se reconoce en el Informe de medio ambiente 2011 que dentro de la agenda conjunta formulada por el MinAgricultura y el MinAmbiente no se dieron avances significativos en ninguno de los cuatro temas estratégicos planteados, incluso el cambio climático. ${ }^{31}$

Además, comentan sobre trabajos colaborativos con el Ideam. Desde Parques Nacionales Naturales de Colombia, mediante malla de variables climatológicas, se espera evaluar y medir el impacto en las áreas protegidas de Santander. Actualmente están levantando información conceptual y teórica para poder visualizar cómo, desde las áreas protegidas, apoyarán el ejercicio de la mitigación. En este momento no se dispone de información suficiente de los ecosistemas en áreas protegidas que permita generar propuestas de adaptación. Refieren sobre estrategias de adaptación, principalmente en áreas protegidas con sistemas productivos, con mayor o menor impacto, que realizan mediante educación a comunidades sin intervención en sus costumbres ancestrales, lo que refleja otro claro ejemplo de estrategias de adaptación al cambio climático.

En cuanto al grupo focal participante en el Foro Regional sobre Cambio Climático convocado por el

31. Contraloría General de la República. INFORME DEL ESTAdo DE LOS RECURSOS NATURALES y DEL AMBIENTE 2011-2012. Contraloría General de la República. (2012). 
Centro de Investigaciones de Medio Ambiente y Desarrollo Sostenible, manifiestan que

para la población no es fácil llevar a cabo estrategias de adaptación al CC [cambio climático], principalmente, cuando la variabilidad climática con elevación de temperatura local obliga a incrementar el consumo de energía tradicional para el enfriamiento de oficinas, viviendas y automóviles, afectando aún más la temperatura del ambiente, por tanto los mecanismos de adaptación al CC, no son los adecuados si no se buscan energías alternativas. ${ }^{32}$

\section{Además, se menciona que}

no hay preparación previa ante fuertes precipitaciones, y la respuesta se da más "acción-reacción”; sin embargo se percibe en la comunidad mayor conocimiento de riesgos potenciales en casos concretos asociado a la presencia de residuos en drenajes y canales que se podrían desviar y desbordar en periodos de precipitaciones fuertes. ${ }^{33}$

Lo anterior refleja capacidad de adaptación, ya que la comunidad se anticipa a los efectos de un disturbio y, para el caso concreto, evita oportunamente arrojar residuos a estos drenajes. Sin embargo, se percibe falta de apropiación de la gestión del riesgo y deficiencias en educación ambiental para la ciudadanía, a pesar de existir un plan estratégico de acción, educación, formación y sensibilización a públicos, que incluye lineamientos a la comunidad en general, autoridad ambiental, tomadores de decisiones, organizaciones específicas, entre otros, los cuales están claramente trazados en la segunda comunicación nacional de Colombia ante la Convención Marco de las Naciones Unidas sobre Cambio Climático.

En cuanto a conocimiento sobre riesgos potenciales e impactos actuales, estos deben ser incorporados en las políticas locales, incluyendo valoración económica, como lo plantea el Plan Nacional de Adaptación al Cambio Climático (PNCC) en sus insumos metodológicos. Además, el Consejo Municipal para la Gestión de Riesgo de Desastre (CMGRD) y la Administración municipal de Norte de Santander han implementado reservorios estratégicos para atender periodos de sequía. Afirman que actualmente han construido 8 de 12 programados, lo que se traduce en un claro ejemplo de adaptación a las amenazas relacionadas con la variabilidad climática asociada a sequías.

Finalmente, los participantes recomiendan, para que la región pueda contar con mayores posibilidades de adaptación al cambio climático, fortalecer la educación ambiental en todas las etapas de formación, contar con los aportes de universidades en investigación y divulgación sobre la temática, además se considera que el cambio climático debe tomarse como un tema obligado y no de moda.

\section{Conclusiones}

A pesar de los esfuerzos del orden central, materializados en políticas nacionales e instrumentos de cambio climático y adaptación a este, sustentadas en estudios previos, circunstancias nacionales con amplio acervo, los gobiernos locales no han logrado del todo articular sus acciones a partir de una planificación integral; sin embargo, la mayoría afirma que existe articulación de políticas relacionadas con el cambio climático establecidas en el рот о Plan de Desarrollo Local.

No se evidencia una clara apropiación de conocimiento sobre cambio climático en los gobiernos locales, por ende, no se percibe la existencia de capacidades para proponer medidas de adaptación y mejorar las capacidades de reacción frente a los impactos del cambio climático, a pesar de existir, desde el orden nacional, medidas de educación sobre este en diferentes actores gubernamentales y la comunidad en general.

Se evidencia, en algunos casos, apropiación, esfuerzos focales y aislados, principalmente liderados por instituciones ambientales y organizaciones no gubernamentales, atendiendo a los lineamientos que, desde el marco nacional, se han venido formulando en los últimos años; sin embargo, falta articulación de estas iniciativas con los entes territoriales.

En general, los diferentes actores identifican las razones desde lo social, económico y ambiental para abordar el cambio climático, coincidentes con las que el Gobierno expone sobre adaptación al cambio climático.

A pesar de identificar la Política Nacional de Gestión de Riesgo de Desastres, aún falta claridad para asumir la gestión del riesgo como una medida de adaptación al cambio climático, lo que se traduce en debilidad en la incorporación de esta política en lo local.

32. Informante 1, grupo focal institucional.

33. Informante 2, grupo focal institucional. 


\section{REFERENCIAS}

Carlos Costa Posada. (Noviembre de 2007). La adaptación al cambio climático en Colombia. RevisTA DE INGENIERías 26, Noviembre del 2007. Pp. 74-80. [Recuperado el 12 de agosto de 2013 de http://www.scielo.org.co/pdf/ ring/n26/n26a10.pdf]

Contraloría General de la República. Informe del estado DE LOS RECURSOS NATURALES Y DEL AMBIENTE 20112012. Contraloría General de la República. (2012).

Departamento Nacional de Planeación. Documento ConPES 3700. Estrategia INSTITUCIONAL PARA LA ARTICULACIÓN DE POLÍTICAS Y ACCIONES EN MATERIA DE CAMBio Climático en COLOMBia. (2011).

Departamento Nacional de Planeación. Plan Nacional de Adaptación al Cambio Climático: Lineamientos y AGENDA PARA LA ADAPTACIÓN AL CAMBIO CLIMÁTICO eN Colombia. Departamento Nacional de Planeación. (2011).

Gobernación de Norte de Santander. Información general: norte de Santander. [En línea] Disponible en http:// www.nortedesantander.gov.co/infgeneral.php (Mayo 24 de 2012).

Grupo Intergubernamental de Expertos sobre el Cambio Climático (IPCC). SEgUNDA EVALUACIÓN: CAMBio CLIMÁTICO. PNUMA. (1995).

Grupo Intergubernamental de Expertos Sobre el Cambio Climático (IPCC). CAMBIO CLIMÁTICO 2001: INFORME DE SÍNTESIS. RESUMEN PARA RESPONSABLES DE POLÍTICAS. (2001).

Grupo Intergubernamental de Expertos Sobre el Cambio Climático (IPCC). Anexo B: glosario de términos. CAMBIO CLIMÁTICO 2001: INFORME DE SÍNTESIS. RESUMEN para RESPONSABles DE POlíticas. (2001). [En línea]. Disponible en: http://www.ipcc.ch/ pdf/glossary/taripcc-terms-sp.pdf
Grupo Intergubernamental de Expertos sobre el Cambio Climático (IPCC). CAMBIO CLIMÁTICO 2007: INFORME DE SÍNTESIS. OMM Y PNUMA. (2008).

Gustavo Wilches-Chaux. EsE OCÉANO DE AIRE EN QUE VIVIMOS. ORIGEN, EVOLUCIÓN, ESTADO ACTUAL Y FUTUROS POSIBLES DE LA ATMÓSFERA TERRESTRE. La Silueta Ediciones. (2008).

Ley 1523 de 2012. Por la cual se adopta la política nacional de gestión del riesgo de desastres y se establece el Sistema Nacional de Gestión del Riesgo de Desastres y se dictan otras disposiciones. Abril 24 de 2012. DO N. ${ }^{\circ} 48411$.

María Carolina Pinilla, Andrés Rueda, Carlos Pinzón y Javier Sánchez. Percepciones sobre los fenómenos de variabilidad climática y cambio climático entre campesinos del centro de Santander, Colombia. Ambiente y DesarroLLO 31. Diciembre de 2012. At. 25.

Michael Comstock, Ignacio Santelices y Anmol Vanamali. CAso DE ESTUDIO: PROCESO NACIONAL DE CAMBIO CLIMÁTICO en Colombia. Center for Clean Air Policy. (2012).

Ministerio de Ambiente y Desarrollo Sostenible, IdeAm \& Embajada de Holanda. El ABC del cambio climático en Colombia. (s. f.). Disponible en: http://www.ideab-ufps. com/nodo/doc/nuevos/abc\%20del\%20cambio\%20climatico\%20en\%20Colombia.pdf

Naciones Unidas. Convención Marco de las Naciones Unidas para el Cambio Climático. (1992). Disponible en: http://unfccc.int/resource/docs/convkp/ convsp.pdf

Oficina Asesora de Planeación. Informe DE GeSTión 2011. Ministerio de Ambiente y Desarrollo Sostenible. (2012).

Ordenanza 08 de 2008 [Gobernación de Santander]. Por la cual se adiciona el presupuesto general de ingresos y gastos del departamento de Santander. Mayo 30 de 2008.

http://www.secretariasenado.gov.co.

http://www.cambioclimatico.gov.co/jsp/1276 (Noviembre de 2012). 